\title{
EL PROCESO DE APRENDIZAJE DEL INDIVIDUO EN EL CONTEXTO ORGANIZACIONAL
}

\section{ARTÍCULO ORIGINAL}

SILVA, Lilian Reis da ${ }^{1}$

SILVA, Lilian Reis da. El proceso de aprendizaje del individuo en el contexto organizacional. Revista Científica Multidisciplinar Núcleo do Conhecimento. Año. 06, Ed. 10, Vol. 08, págs. 17-30. Octubre de 2021. ISSN: 2448-0959, Enlace de acceso: https://www.nucleodoconhecimento.com.br/educacion-es/aprendizaje-delindividuo, DOI: 10.32749/nucleodoconhecimento.com.br/educacion-es/aprendizajedel-individuo

\section{RESUMEN}

Este artículo, elaborado a través de la investigación bibliográfica, tiene como cuestión fundamental la forma en que los individuos aprenden e interactúan en el contexto de las organizaciones. Con el objetivo de abordar cómo el aprendizaje individual contribuye al aprendizaje organizacional entre los individuos. De la literatura analizada, se encontró que los conocimientos y conocimientos adquiridos individualmente por las personas deben sumarse a las habilidades de comunicación, interacción y compartir con los colegas, dentro del ámbito de las organizaciones, para que juntos aprendan la cultura, el sistema interno, los objetivos y las prácticas inherentes al mismo, con el fin de alcanzar las metas existentes.

Palabras clave: Capital Humano, Conocimientos, Habilidades, Desempeño Profesional, Aprendizaje Organizacional.

\footnotetext{
${ }^{1}$ Postgrado en Dirección de Empresas, Economista, Técnica Contable.
}

RC: 100494

Disponible: https://www.nucleodoconhecimento.com.br/educacion-es/aprendizajedel-individuo 


\section{INTRODUCCIÓN}

A lo largo de su vida profesional, todas las personas tienen innumerables oportunidades de aprendizaje más allá de lo convencional - e individual - como, por ejemplo, nuevas formas de trabajar, que tienden a ser diferentes de una empresa a otra. Este aprendizaje también puede darse a partir de nuevas lecturas, cursos de especialización o la identificación de videos en Internet, sobre temas inherentes al área de especialización. También existe la creación de redes en sí.

Dado el volumen de información y modernizaciones que vive la sociedad global, Bezerra y Oliveira (2006, s.p.) se refieren a Guns (1998, p.7) para confirmar que el volumen de información hoy en día es tan grande que "los ejecutivos, gerentes y trabajadores de primera línea también se enfrentan a cantidades gigantescas de información".

A su vez, la valorización de los individuos en una sociedad que hoy ya reconoce que el capital humano es el activo más preciado dentro de las organizaciones (CHIAVENATO, 2020), los trabajadores dejaron ese rol de simples ejecutores de tareas previamente definidas, pasando al rol de clientes internos, quienes requieren actualizaciones constantes para desempeñar mejor sus funciones, cuyos resultados deben cumplir con las metas establecidas por la dirección de dichas organizaciones.

Esta realidad, no tan reciente - trae a todos el concepto sobre el aprendizaje organizacional, que, según Guns (1998, p. 33, apud BEZERRA y OLIVEIRA, 2006, s.p.), consiste en adquirir "conocimientos, habilidades, valores, convicciones y actitudes que aumentan el mantenimiento, crecimiento y desarrollo de la organización".

Por lo tanto, el objetivo de este artículo es abordar cómo el aprendizaje individual contribuye al aprendizaje organizacional entre los individuos.

RC: 100494

Disponible: https://www.nucleodoconhecimento.com.br/educacion-es/aprendizajedel-individuo 


\section{LOS PROCESOS DE APRENDIZAJE DE LOS INDIVIDUOS}

El aprendizaje consiste en el acto del individuo de adquirir conocimiento, ya que "es la naturaleza del conocimiento que cambia rápidamente y que las certezas de hoy se convierten en los absurdos de mañana" (DRUCKER, 1999, p.121).

Aprender en un mundo en constante cambio no es algo fácil, y es esencial, en los tiempos modernos, que los profesionales de todas las áreas sigan aprendiendo. Fleury y Fleury (2001, apud MENEZES et al., sin fecha, p. 2) enseñan que "el aprendizaje se considera como un proceso de cambio, desencadenado por diversos estímulos, mediados por emociones, que pueden o no manifestarse en el comportamiento cambiante del individuo".

Según el Diccionario Houaiss de la lengua portuguesa (HOUAISS, 2015, p. 74), el aprendizaje es "acto, duración y experiencia, acción de aprender un oficio o profesión" y aprender es "adquirir conocimientos prácticos, tener una mejor comprensión de (algo), por intuición, experiencia o convivencia [...]".

A Bruni; Turrioni y Stano (2005, p.190), es un proceso psicológico que es fundamental para la supervivencia humana, que le permite adaptarse al entorno en el que vive, e incluso conquistar el crecimiento moral, intelectual y profesional. Menezes et al. (sin fecha, p.2) informe Abbad y Borges-Andrade (2004, p.238), para destacar que "el aprendizaje es un proceso psicológico que ocurre a nivel individual.

Desde la perspectiva de Julius (2003), la gran pregunta relacionada con el aprendizaje es la necesidad de que las personas aprendan a aprender, para que siempre puedan aprender. Sucede que para aprender los individuos buscan el conocimiento a través de la información, lo que, a su vez, permite a las personas alcanzar nuevos conocimientos a través de la elección, selección, asimilación y asociación, con el objetivo de mejorar y aprender a aprender. El hecho es que mientras el conocimiento es el resultado de la selección de información, que se

RC: 100494

Disponible: https://www.nucleodoconhecimento.com.br/educacion-es/aprendizajedel-individuo 
acumula, es decir, el conocimiento de una persona se construye a través de lo que elige en base a la información que obtiene y que confía en ella (JULIO, 2003).

A estos hechos se suma la aparición de Internet, que permite a los individuos obtener los más diferentes contenidos e información, incluso en tiempo real, aunque cada vez ha sido más común que se localizan muchos materiales que no se corresponden con la realidad y, por lo tanto, no logran agregar ningún valor a los usuarios de Internet.

Según Bruni et al. (2005, p.190), el aprendizaje ocurre efectivamente desde el momento en que el individuo pasa por una determinada experiencia, ya que las numerosas conexiones cerebrales que ocurren permiten a la persona asociar los hechos que sucedieron en su entorno.

En esta perspectiva, Senge (2002, p.32) sostiene que "todo aprendizaje está relacionado con la acción. El aprendizaje nunca ocurre exclusivamente a través del estudio pasivo". Still Bruni et al. (2005) explican que es a través de la interacción entre las personas que el conocimiento se expande, individual y colectivamente. Otro aspecto relevante destacado por estos autores son los tipos de aprendizaje que añaden conocimiento a los individuos, son: el aprendizaje formal o multidisciplinario, y el aprendizaje informal, que tiene lugar en todos los lugares y diferentes situaciones, a partir de la observación de las circunstancias y la interacción entre las personas.

En este sentido, Menezes et al. (sin fecha, p.4) informan que el aprendizaje formal se lleva a cabo en base a acciones específicas respecto a las tareas y objetivos a cumplir, relatando esencialmente "técnicas, métodos, condiciones y lugares especificados anteriormente y que se pretende originar conocimientos, actitudes, habilidades, comportamientos e ideas". También según los autores, el aprendizaje formal de los individuos se elabora bajo un método que contiene estructuras organizadas.

RC: 100494

Disponible: https://www.nucleodoconhecimento.com.br/educacion-es/aprendizajedel-individuo 
A su vez, el aprendizaje informal es, aunque la relevancia del aprendizaje formal para los individuos, en el contexto de su vida personal, desde la perspectiva del entorno organizacional, es indiscutible, las diferentes oportunidades y objetivos de cada empresa requerirán, en sí mismo, que sus empleados tengan diferentes habilidades y conocimientos, adquiridos en actividades prácticas, como Flach y Antonello (2010, apud MENEZES et al., sin fecha, p. 4).

En este sentido, Menezes et al. (sin fecha, p.4) informe Zerbini (2007), para argumentar que el aprendizaje informal consiste en procesos por los cuales las personas observan conversaciones formales o informales, ya sea en reuniones de trabajo o en ambientes relajados.

De lo contrario, el aprendizaje informal tiene lugar cuando una persona tiene - por iniciativa propia y para su crecimiento personal y profesional - la posibilidad de aprender nuevas visiones y actitudes, lo que le permitirá marcar la diferencia en su desempeño profesional. Se considera que "ocurre fuera de los planes de estudio de cursos, programas educativos $o$ talleres" (COELHO JÚNIOR y BORGES ANDRADE, 2008, apud MENEZES et al., s. d., p.4).

Otro proceso en la forma de aprender a aprender es el reconocimiento de las diferencias entre las personas y el respeto por otras experiencias de vida que se registran en sus mentes, que influyen directamente en el comportamiento de estas personas.

En este sentido, Romanowski y Peranzoni (2011) informan sobre la importancia de que a los niños se les enseñe, desde el principio, a aceptar las diferencias entre las personas, en la forma en que son, piensan y actúan; a los niños se les debe enseñar a respetar la diversidad, desde una edad temprana, hasta la edad adulta, e incluso profesional.

RC: 100494

Disponible: https://www.nucleodoconhecimento.com.br/educacion-es/aprendizajedel-individuo 
La importancia de que cada individuo aprenda y reconozca las diferencias entre las personas radica en el hecho de que, en las regiones más variadas del país o del mundo, hay diferencias en la educación y la capacitación recibida por los individuos en el hogar o la escuela, los hábitos difieren, la visión sobre la vida y sobre las situaciones y las personas, difiere la orientación sexual, y muchos otros aspectos son diferentes, de un individuo a otro (ROMANOWSKI y PERANZONI (2011).

Esta realidad requiere que las diferencias en la percepción, el aprendizaje y el comportamiento de las diferentes personas sean aceptadas por quienes las rodean, siendo muy importantes para la vida y la estrategia empresarial, porque la buena relación y el respeto conducen a una mayor productividad y competitividad en el entorno empresarial.

En este sentido, todavía Romanowski y Peranzoni (2011, s.p.) informe Perreoud (2000) para afirmar que para aprender algo nuevo, cada persona necesita ser colocada en situaciones de aprendizaje con frecuencia; además, que estas situaciones tengan sentido para este alumno, y que pueda sentir y movilizarse con y para ellos.

\section{3. ¿CÓMO DESARROLLAR EL PROCESO DE APRENDIZAJE FRENTE A LAS HABILIDADES ORGANIZATIVAS?}

\subsection{ENTRE LÍNEAS}

Todas las organizaciones,independientemente de su estructura o segmento- tienen una cultura organizacional que, según Chiavenato (2010), consiste en "hábitos y creencias, establecidos por normas, valores, actitudes y expectativas, compartidas por todos los miembros de la organización. Se refiere al sistema de significados compartidos por todos los miembros y que distingue a una organización de la otra".

RC: 100494

Disponible: https://www.nucleodoconhecimento.com.br/educacion-es/aprendizajedel-individuo 
En este sentido, Wilbert y Cruz (2014, p. 2), enseñan que para que una empresa sea efectiva en su segmento de actividad, es fundamental que el pensamiento, los valores y las acciones adoptadas en su contexto estén alineados con los diferentes tipos de desafíos diarios, permitiendo el logro de los resultados esperados.

Con el tiempo, especialistas como Chanlat (1998) han investigado el comportamiento humano, que no se trata solo de lo que es observable, ya que también se debe a otros aspectos, como el deseo, el deseo y el impulso del individuo. Del mismo modo, investigadores especializados en gestión organizacional elaboraron análisis conductuales en organizaciones, cuyo interés era observar los fenómenos humanos desde una perspectiva de cultura psicoanalítica, sugiriendo tres líneas de visión, que fueron votadas por Chanlat (1998) y otros autores:

a) linha de visão do grupo: vista como um sistema social de defesa contra a angústia e depressão, que analisa o comportamento despersonificado que até hoje inspira numerosos trabalhos (CHANLAT, 1998 apud JAQUES);

b) linha de visão do dirigente: uma segunda linha de pesquisa que procura destacar não apenas o papel e a importância que o imaginário exerce nas ações dos dirigentes das empresas, mas também as conseqüências de sua incidência sobre as organizações (CHANLAT, 1998 apud LEVINSON);

c) linha de visão da organização: uma linha que possui uma abordagem na psicologia social, onde vê a relação homem versus conflitos, elucidando até hoje o que - na organização do trabalho - entra em conflito com o funcionamento do aparelho psíquico (CHANLAT, 1998 apud DEJOURS).

Estos trabajos apuntan a la necesidad de tomar conciencia para entender la vida organizacional, una importante herramienta de gestión. Es a través de este aprendizaje que podemos valorar la vida interior y el afecto que están presentes en la vida organizacional y cuánto las relaciones humanas están marcadas fundamentalmente por necesidades y voluntades (SOUZA, 2016).

RC: 100494

Disponible: https://www.nucleodoconhecimento.com.br/educacion-es/aprendizajedel-individuo 


\subsection{COMPETENCIA}

La búsqueda del aprendizaje en las organizaciones adaptando sus conocimientos, su capacidad para una actividad determinada y su poder de decisión son los elementos necesarios para aumentar las habilidades de los empleados en las organizaciones, ya que, para cada puesto asumido, es necesario que todo el proceso de aprendizaje sea muy claro, permitiendo al trabajador un buen desempeño, y resultados dentro de lo esperado (CROZATTI, 1998).

Según el Mini diccionario Aurélio de la lengua portuguesa (FERREIRA, 2008, p. 249), el término competencia se define como: "capacidad para resolver cualquier tema, aptitud, idoneidad". A partir de esta definición, se observa que la competencia revela su poder cuando se aprovecha, es decir, se aprende, se entiende y se comprende, y cuando hay transformaciones del mundo del trabajo, ya sea en las empresas o en la sociedad.

Definir los temas individuales necesarios para un buen desempeño en cada ocupación no es suficiente para que se produzcan innovaciones o se desarrollen nuevas habilidades para las empresas. El puesto y la ocupación definen la cualificación, o el conocimiento o conocimiento de cada individuo puede ser clasificado por el sistema educativo. El concepto de competencia busca el camino de la proactividad, estando siempre por delante anticipando y dominando lo que está sucediendo y lo que está por venir, no se limita, por tanto, a un abano de conocimiento teórico y empírico en poder del individuo, ya que no está implícito en la tarea (EBERT; POSSAMAI Y SIMÓN, 2017).

Viendo la competencia como inteligencia práctica del conocimiento adquirido y transformado, surge un nuevo estándar de competencia, presentado por Fleury y Oliveira Júnior (2001, apud ZARIFIAN, 2001):

a) necessidade de se antecipar aos fatos e às situações: as quais podem - de alguma forma - tumultuar o bom andamento das

RC: 100494

Disponible: https://www.nucleodoconhecimento.com.br/educacion-es/aprendizajedel-individuo 
atividades e a capacidade de resolver as situações e problemas, para garantir o bom andamento do trabalho;

b) necessidade de comunicação e entendimento: do objetivo organizacional usando de empatia, para $o$ alinhamento $e$ partilhamento da cultura organizacional e cumprimento das normas da empresa. Neste sentido, Jean François Chanlat (1998) explica que a comunicação é absolutamente necessária, significando inclusive que - na maior parte do tempo - significa transmitir informação, sendo possível essa comunicação de várias maneiras: por meio de atitudes, de gestos, de modos de vestir, por meio de objetos colocados à nossa volta ou que manipulamos, de espaços organizados etc.;

c) noção de um serviço: entender um cliente independente de ser um cliente interno ou externo sempre precisa ser o foco central das atividades e para que isto ocorra, se faz fundamental a comunicação.

Dentro de una organización, el trabajo deja de ser un conjunto de tareas y se convierte en la suma de las habilidades y competencias utilizadas para resolver diversas situaciones, ya que son habilidades profesionales cada vez más rentables y complejas. Esta complejidad de situaciones hace que lo imprevisto sea cada vez más común en el día a día (LIMA; ZAMBRONI-DE-SOUZA y ARAÚJO, 2015).

Al pensar en la organización y su totalidad y no sólo en las actividades de producción, el foco principal del análisis (FLEURY y OLIVEIRA JÚNIOR, 2001, apud ZARIFIAN, 2001) observamos la necesidad de introducir otras dos menciones relacionadas con mutaciones en el mundo del trabajo:

a) âmbito de atuação da organização: local, regional, nacional ou global: as mudanças de operações da empresa, nesses últimos anos em função do processo de globalização têm implicações significativas para a localização e a formação de competências não só em termos da organização. Percebe-se que essa visão está em todos os níveis (com diferentes ponderações e significações), fazendo parte da competência individual;

b) antes da visão estratégica: ficava no topo das organizações, mas atualmente deve estar em diferentes níveis com significação e critério distintos, compondo o perfil da competência individual.

RC: 100494

Disponible: https://www.nucleodoconhecimento.com.br/educacion-es/aprendizajedel-individuo 
La competencia del individuo no es un estado, no es sólo un conocimiento exacto, sino que lo sitúa como resultado del cruce de tres ejes: la formación de la persona (su biografía y socialización), su formación educativa y su experiencia profesional (FLEURY y FLEURY, 2004, apud LE BOTERF, 1997).

La competencia es una forma responsable de actuar con tal reconocimiento por parte de los demás. Surge como: saber aprender, saber comprometerse, asumir responsabilidades, tener una visión estratégica, agregar valor económico a la organización y valor social para el individuo.

Las competencias generan valor económico para las organizaciones, así como el debido valor social para el individuo; así, podemos decir que la competencia organizacional es un portafolio de competencias, físicas (infraestructura), financieras, intangibles, corporativas (recursos humanos), siendo esta última lo que creemos que es el gran diferencial que crea ventajas competitivas y de esta manera, debemos contar con estrategias competitivas dadas por estos recursos. Según Fleury y Fleury (2004), "un recurso es algo a lo que la organización tiene o tiene acceso, incluso si este acceso es temporal, [...] una competencia se construye a partir de un conjunto de 'bloques' llamados recursos".

La competencia es la inteligencia de conciliar, mezclar e incluir recursos en productos y servicios. La competitividad de una organización estaría plenamente ligada en la interacción entre competencia organizacional y estrategia competitiva de esta manera, el enfoque de recursos hace que el proceso de formulación de la estrategia y la formación de competencias formen un círculo que se retroalimenta (FLEURY y FLEURY, 2004).

Las competencias básicas ofrecen beneficios reales a los consumidores, beneficios difíciles de imitar, y que dan acceso a diferentes mercados, y cuando la empresa define su estrategia competitiva, termina identificando sus competencias

RC: 100494

Disponible: https://www.nucleodoconhecimento.com.br/educacion-es/aprendizajedel-individuo 
empresariales esenciales y las competencias necesarias de cada función habilidades organizativas (FLEURY y FLEURY, 2004).

Desde esta perspectiva, se verifica que las empresas de diversas competencias organizacionales (competencias colectivas asociadas a actividades intermedias y actividades finales) en varias áreas; sin embargo, solo unas pocas son las competencias esenciales, es decir, son las que las diferencian y garantizan una ventaja competitiva sostenible frente a otras organizaciones. Para ser considerada una competencia esencial, debe estar directamente vinculada a los procesos de aprendizaje organizacional, lo que da enfoque y reposiciona las estrategias competitivas (FLEURY y FLEURY, 2004).

Hay tres tipos de estrategia que pueden ser adoptadas por las organizaciones, a través de las cuales las empresas compiten en el mercado, según Fleury y Fleury (2004):

a) excelência operacional: empresas que competem baseadas no custo, oferecendo aos seus clientes produtos com o melhor preço e atendimento adequado;

b) inovação no produto: empresas que oferecerem aos seus clientes produtos de ponta, inovando sempre;

c) orientada para clientes: característica de empresas voltadas a atender a demanda de clientes específicos, e antecipar as necessidades desses clientes, em função de sua proximidade com os mesmos.

Una vez definida la estrategia competitiva, la empresa es capaz de identificar las competencias básicas del negocio, así como las habilidades necesarias para cada función. Esta práctica permite a su junta directiva pasar del nivel estratégico al nivel de formación de las competencias del individuo, competencias categorizadas por Fleury y Oliveira Júnior (2001) en tres grandes bloques que involucran la relación del individuo con toda la organización:

RC: 100494

Disponible: https://www.nucleodoconhecimento.com.br/educacion-es/aprendizajedel-individuo 
a) competências de negócio: relacionadas ao negócio em si, seu mercado, clientes, concorrentes;

b) competências técnico-profissionais: habilidades específicas para a realização de determinada operação;

c) competências sociais: competências necessárias para interagir com as pessoas, entre elas a competência interdisciplinar necessárias a acordos, à comunicação, à liderança, e ao respeito às culturas distintas.

La comunicación es de suma importancia porque asegura la comprensión de los mensajes por parte de los otros miembros de la organización; consiste en la capacidad de expresar ideas con claridad, utilizando técnicas de comunicación adecuadas a cada situación (CHIAVENATO, 2000).

A través de la comunicación, se debe tener la capacidad de discutir, estimular e influir positivamente en otras personas para que colaboren, de manera efectiva, de manera que se logren los objetivos organizacionales, realizando procesos de consenso, enfocándose en resultados satisfactorios para las partes involucradas y, principalmente, para la organización. Tales herramientas son válidas para situaciones externas e internas, como la forma ideal de argumentar, de modo que permite a las personas persuasión en la venta de ideas y aceptar puntos de vista divergentes desde su visión inicial (CROZATTI, 1998).

El trabajo en equipo debe crear y trabajar con grupos de personas que tengan habilidades y conocimientos diversos y complementarios, proporcionando el surgimiento de sinergia, que es la capacidad de mantener a dichos grupos trabajando juntos de manera eficiente, controlando las participaciones excesivas e invitando a miembros silenciosos a participar, resolviendo conflictos (CHIAVENATO, 2000).

Las discusiones sobre el aprendizaje en las organizaciones se basan más fuertemente en la perspectiva intelectual, evidenciando cambios de comportamiento. La forma en que las personas manifiestan y desarrollan las competencias

RC: 100494

Disponible: https://www.nucleodoconhecimento.com.br/educacion-es/aprendizajedel-individuo 
fundamentales dentro de la organización y su proyecto profesional, es algo que ocurre solo a través del proceso de desarrollo de sus competencias (CHIAVENATO, 2000). Es un conjunto de situaciones de aprendizaje que pueden llevar a la transformación del aprendizaje en competencias; como resultado, la suma de valor ocurrirá tanto para la persona como para la organización.

Es un proceso que puede ocurrir a diferentes niveles, como destacan Fleury y Fleury (2004):

a) ao nível individual: que é onde ocorre primeiro, e como já citado, em um indivíduo que é carregado de emoções positivas ou negativas, por meio dos caminhos que percorreu;

b) ao nível grupal: onde a aprendizagem pode vir a se constituir em um processo social e coletivo, sendo que para compreendê-lo é importante entender como é o aprendizado do grupo, como são integradas as crenças individuais com o grupo e, como transformam esse compartilhamento em ações, além do desejo de pertencer ao grupo, que pode constituir um elemento motivacional ao processo de aprendizagem;

c) em nível organizacional: o processo de aprendizagem individual, de compreensão e interpretação compartilhados pelo grupo, torna-se institucionalizado e conhecido em vários mecanismos organizacionais, ou seja, estrutura, regras, procedimentos, e até mesmo em elementos simbólicos, já que, assim como o indivíduo, as organizações também expandem as memórias que possuem informações e as recuperam.

En esta etapa, vale la pena analizar los procesos de aprendizaje. Con respecto al proceso de aprendizaje organizacional, es el tipo que implica la elaboración de mapas de conocimiento, que permiten comprender lo que ocurre en el entorno externo e interno de la organización, así como en la determinación de nuevos procedimientos, que demuestran la efectividad del aprendizaje (FLEURY y FLEURY, 2004).

Para ello, las organizaciones confían en sus sistemas de conocimiento y memoria, lo que les permite desarrollar rutinas y procedimientos para hacer frente a los

RC: 100494

Disponible: https://www.nucleodoconhecimento.com.br/educacion-es/aprendizajedel-individuo 
problemas. Tales procedimientos se incorporan visiblemente a la memoria organizacional.

El cambio en el proceso, las estructuras o los procedimientos son indicadores de que se produjo el aprendizaje, lo que se suma a la facilidad con la que estos conocimientos son recuperados por los miembros de la organización. Según Fleury y Fleury (2000), "es el conocimiento tácito, a veces inconsciente, el que descansa en la memoria organizacional". En este sentido, el proceso de aprendizaje tiene muchas facetas:

a) "Aprendizagem organizacional é um processo de identificação e correção de erros" (ARGYRIS, 1997, p.116 apud FLEURY e FLEURY, 2000, p. 9);

b) "Aprendizagem organizacional significa um processo de aperfeiçoar as ações pelo melhor conhecimento e compreensão" (FIOL E LYLES, 1985, p.803 apud FLEURY e FLEURY, 2000, p. 9);

c) "organizações que aprendem são organizações capacitadas em criar, adquirir e transferir conhecimentos e modificar seus comportamentos para refletir esses novos conhecimentos e 'insights'" (GARVIN, 1993, p.80 apud FLEURY e FLEURY, 2000, p.9);

d) "Uma organização está continuamente expandindo sua capacidade de criar o futuro" (SENGE, 1990, p.14 apud FLEURY e FLEURY, 2000, p.9).

Una organización aprende cuando se acelera su capacidad de adaptación al cambio, constituyendo el primer paso en el proceso de aprendizaje. El deseo de aprender va más allá: es innovador y fructífero.

\section{CONCLUSIÓN}

A través del análisis de las teorías seleccionadas para la elaboración de este estudio, se verifica que los procesos de aprendizaje involucran diferentes aspectos, tanto personales como profesionales, por lo que el aprendizaje dentro de las organizaciones se desarrolla dentro de las perspectivas de su dirección.

RC: 100494

Disponible: https://www.nucleodoconhecimento.com.br/educacion-es/aprendizajedel-individuo 
$\mathrm{Si}$, por un lado, las experiencias personales, la educación formal y las características de la personalidad de los individuos colaboran para su formación personal, son elementos que se sumarán a las características, pautas y sistemas de trabajo existentes dentro de las empresas, y es necesario que cada trabajador (a) se adapte de la mejor manera, además de identificar las existencias de posibilidades de expansión de sus habilidades y creatividad -o no- según las expectativas organizacionales.

\section{REFERENCIAS}

BEZERRA, Alan André Aparecido; OLIVEIRA, Ednilson Barbosa de. Aprendizagem Organizacional: a organização que aprende. Artigo publicado em 06 jul 2006. Disponível em: [https://administradores.com.br/artigos/aprendizagem-organizacionala-organizacao-que-aprende]; acesso em 28 mar 2021.

BRUNI, Márcia; TURRIONI, João Batista; STANO, Rita de Cássia Trindade. Abordagens da aprendizagem no contexto organizacional. II Simpósio de Excelência em Gestão e Tecnologia - SEGeT'2005. p. 190-198. Disponível em: [https://www.aedb.br/seget/arquivos/artigos05/274 Abordagens\%20da\%20aprendiza gem\%20no\%20contexto\%20organizacional.pdf]; acesso em 28 mar 2021.

CHANLAT, Jean François. 0 indivíduo na Organização. 1.ed. São Paulo: Atlas, 1998, v.3.

CHIAVENATO, Idalberto. Recursos humanos - 0 capital humano das organizações. 11.ed. São Paulo: Atlas, 2020.

Gestão de pessoas: o novo papel dos recursos humanos nas organizações. 3.ed. Rio de Janeiro: Elsevier, 2010.

CROZATTI, Jaime. Modelo de Gestão e Cultura Organizacional - conceitos e interações. Caderno de Estudos, SP, FIPECAFI, vol. 10, n. 18, maio/ago 1998.

RC: 100494

Disponible: https://www.nucleodoconhecimento.com.br/educacion-es/aprendizajedel-individuo 
DRUCKER, Peter F. Administrando em Tempos de Grandes Mudanças. São Paulo: Pioneira, 1999.

EBERT, Luis Augusto; POSSAMAI, Cleide Tirana Nunes; SIMON, Vanessa Silveira. Perspectivas profissionais. Indaial: UNIASSELVI, 2017. Disponível em: [https://www.uniasselvi.com.br/extranet/layout/request/trilha/materiais/livro/livro.php? codigo=25048]; acesso em 03 abr 2021.

FERREIRA, Aurélio Buarque de Holanda. Miniaurélio: o minidicionário da língua portuguesa. 7.ed. Curitiba: Positivo, 2008.

FLEURY, Afonso C. C.; FLEURY, Maria Tereza Leme. Estratégias Competitivas E Competências Essenciais: Perspectivas Para A Internacionalização Da Indústria No Brasil. Gestão \& Produção, vol.10, n.2, p.129-144, ago. 2003. Disponível em: [https://www.scielo.br/i/gp/a/C8hMY4qXzCfGWd4v8VRwXJQ/?lang=pt]; acesso em 28 mar 2021.

FLEURY, M. Teresa Keme; OLIVEIRA JR., Moacir de M. (Coord.). Gestão estratégica do conhecimento: integrando aprendizagem, conhecimento e competências. São Paulo: Atlas, 2001.

GUNS, Bob. A organização que aprende rápido: seja competitivo utilizando o aprendizado organizacional. São Paulo: Futura, 1998.

HOUAISS, Antonio; VILLAR, Mauro de Salles; FRANCO, Francisco Manoel de Mello. Pequeno Dicionário Houaiss da Língua Portuguesa. 1.ed. São Paulo: Moderna, 2015.

JULIO, Carlos Alberto. A Magia dos Grandes Negociadores. 1.ed. Rio de Janeiro: Elsevier, 2003.

RC: 100494

Disponible: https://www.nucleodoconhecimento.com.br/educacion-es/aprendizajedel-individuo 
LIMA, Claudia Maria Pereira de; ZAMBRONI-DE-SOUZA, Paulo César; ARAÚJO, Anísio José da Silva. A Gestão do Trabalho e os Desafios da Competência: uma Contribuição de Philippe Zarifian. Psicologia: Ciência e Profissão, vol. 35, n. 4, p. 1223-1238, 2015.

Disponível em: [https://www.scielo.br/j/pcp/a/xxL7S4fWDxwkKWmkB4rtTWp/abstract/?lang=pt]; acesso em 28 mar 2021.

MENEZES, Bárbara Sampaio de; CABRAL, Augusto Cézar De Aquino; SANTOS, Sandra Maria dos; SILVA, Maria Naiula Monteiro da. Aprendizagem individual no contexto organizacional: percepção dos estudantes da FEAAC - UFC. Artigo publicado nos Anais do ENGEMA - Encontro Internacional sobre Gestão Empresarial e Meio Ambiente. Disponível em: [http://engemausp.submissao.com.br/17/anais/arquivos/81.pdf]; acesso em 28 mar 2021.

PERRENOUD, Philippe. Dez novas competências para ensinar: convite à viagem. Porto Alegre: Artes Médicas, 2000.

ROMANOWSKI, Caroline Leonhardt; PERANZONI, Vaneza Cauduro. Educação para diversidade humana: respeito às diferenças $e$ valorização da singularidade. EFDeportes.com, Revista Digital. Buenos Aires - Año 16 - № 158 julho de 2011. Disponível em: [https://www.efdeportes.com/efd158/educacao-paradiversidade-humana.htm]; acesso em 03 abr 2021.

SENGE, Peter M. A Quinta Disciplina: Arte e Prática da Organização que aprende. 10. ed. São Paulo: Best Seller, 2002.

WILBERT, Alison; CRUZ, Hélio Alves da. Cultura e Clima Organizacionais: uma análise na empresa Novo Trigo de Balneário Camboriu/SC. XI SEGeT - Simpósio de Excelência em Gestão e Tecnologia, de 22 a 24 de outubro de 2014. Disponível

RC: 100494

Disponible: https://www.nucleodoconhecimento.com.br/educacion-es/aprendizajedel-individuo 
em: [https://www.aedb.br/seget/arquivos/artigos14/45120574.pdf]; acesso em 28 mar 2021.

Enviado: Marzo de 2021.

Aprobado: octubre de 2021.

RC: 100494

Disponible: https://www.nucleodoconhecimento.com.br/educacion-es/aprendizajedel-individuo 